\title{
Engineered Coatings for Titanium Implants To Present Ultralow Doses of BMP-7
}

Mohammed Al-Jarsha, ${ }^{\dagger, \|, \Phi l}$ Vladimíra Moulisová, ${ }^{\ddagger}, \mathbb{I} \odot$ Aldo Leal-Egaña, ${ }^{\ddagger}$ Andrew Connell, ${ }^{\S}$ Kurt B. Naudi, $^{\dagger}$ Ashraf F. Ayoub, ${ }^{\dagger}$ Matthew J. Dalby, ${ }^{\ddagger}$ and Manuel Salmerón-Sánchez ${ }^{*} \neq(0)$

${ }^{\dagger}$ Department of Oral and Maxillofacial Surgery, Dental Hospital and School, Glasgow University, G2 3JZ Glasgow, United Kingdom

${ }^{\ddagger}$ The Centre for the Cellular Microenvironment, University of Glasgow, G12 8LT Glasgow, United Kingdom

${ }^{\S}$ Division of Biomedical Engineering, School of Engineering, University of Glasgow, G12 8QQ Glasgow, United Kingdom

"Department of Oral Surgery, College of Dentistry, University of Baghdad, 10001Baghdad, Iraq

Supporting Information

ABSTRACT: The ongoing research to improve the clinical outcome of titanium implants has resulted in the implemetation of multiple approches to deliver osteogenic growth factors accelerating and sustaining osseointegration. Here we show the presentation of human bone morphogenetic protein 7 (BMP-7) adsorbed to titanium discs coated with poly(ethyl acrylate) (PEA). We have previously shown that PEA promotes fibronectin organization into nanonetworks exposing integrinand growth-factor-binding domains, allowing a synergistic interaction at the integrin/growth factor receptor level. Here, titanium discs were coated with PEA and fibronectin and then decorated with $\mathrm{ng} / \mathrm{mL}$ doses of BMP-7. Human mesenchymal stem cells were used to investigate cellular responses on these functionalized microenvironments. Cell adhesion, proliferation, and mineralization, as well as osteogenic markers expression (osteopontin and osteocalcin) revealed the ability of the system to be more potent in osteodifferentiation of the mesenchymal cells than combinations of titanium and BMP-7 in absence of PEA coatings. This work represents a novel strategy to improve the biological activity of titanium implants with BMP-7.

KEYWORDS: bone morphogenetic protein 7, fibronectin network, osteogenic differentiation, poly(ethyl acrylate) (PEA), functionalized titanium

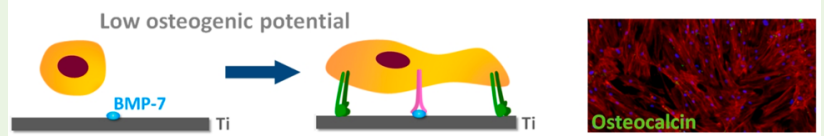

\section{INTRODUCTION}

Bone-healing properties of demineralized bone have been known since the end of 19th century; however, it was not until the 1960s when a seminal paper was published describing the osteoinductive effect of bone morphogenetic proteins (BMPs). ${ }^{1,2}$ Titanium (Ti) provides a high strength and a low elastic modulus, reducing the impact of peri-implant bone resorption. Ti chemistry favors the formation of a biocompatible titanium oxide surface layer, which also imparts increased resistance to corrosion. Therefore, $\mathrm{Ti}$ is often regarded as a more advantageous implant material, when compared with other metals such as cobalt, nickel, chromium, and their respective alloys. ${ }^{3,4}$ The rate of bone formation, the implant surface area directly contacting bone, and the mechanical properties of the bone/implant interface are influenced by the nature of the implant surface. ${ }^{5}$ Previous studies to enhance the surface of $\mathrm{Ti}$ as an implant material were challenged by confounding factors. ${ }^{4}$ These included physical characteristics (e.g., surface roughness) and multiple biologically active factors in the serum when the samples were tested in vitro. 4,6

One option to enhance the biocompatibility of artificial implants is the deposition of extracellular matrix (ECM) proteins onto these surfaces with the purpose to mediate cell-

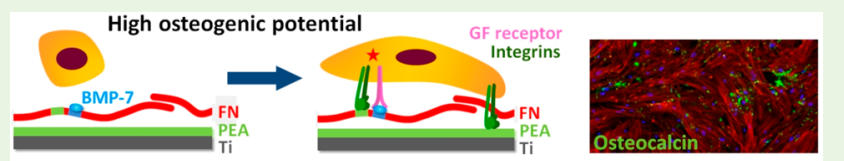

matrix adhesion. Among others, fibronectin (FN) is one of the soluble ECM proteins playing a critical role in osteogenic differentiation. ${ }^{4,7-9}$ It was also found that crucial binding sites for many growth factors on the FN molecule become available when FN is unfolded. It is important to note that these sites are not available when the $\mathrm{FN}$ is in its globular conformation. 7,10

In previous works, we have shown that adsorption of $\mathrm{FN}$ onto poly(ethyl acrylate) (PEA) induced unfolding and then spontaneous organization into nanonetworks, enhancing the availability of both integrin binding domain (FNIII9-10) and the growth factor binding regions (FNIII12-14). ${ }^{7,10}$ The significance of the synergistic effect of integrin and growth factor receptor signaling on PEA/FN substrate has already been reported for the BMP-2-functionalized system, which enhanced osteogenic differentiation of hMSC in vitro. ${ }^{11}$ Moreover, specific VEGF binding to FNIII12-14 on PEA stimulated endothelial cells toward vessel formation. ${ }^{12}$

Bone morphogenetic proteins (BMPs) act as potent regulators during bone and cartilage formation and repair,

Received: January 17, 2018

Accepted: April 22, 2018

Published: April 22, 2018 
mainly through the SMAD pathway. ${ }^{8,13}$ The ability to achieve an early localized continuous expression of BMPs around implants has been the focus of many previous studies. ${ }^{14}$ Recombinant human BMP-2 and BMP-7 have been approved for clinical use in the regeneration of bone for fracture healing and spinal fusion. ${ }^{2}$ Even though BMP-2 has gained much of the focus in the previous literature for bone regeneration, ${ }^{2}$ recent studies have demonstrated the potential of BMP-7 as bone regeneration stimulator. ${ }^{8,15}$ Besides, it has been documented that BMP-7 induces the commitment of undifferentiated nonosteogenic cells into osteoblasts as well as stimulating the maturation of committed osteoblast progenitors. ${ }^{8}$ Finally, BMP-7 has also demonstrated promising bone regenerative capacity in vivo. ${ }^{16}$

Regarding the clinical use of these growth factors (GF), GFs are usually administered by being released from a scaffold or sponge, and it has been shown they can provide significant osseoinductive signals. ${ }^{2}$ However, the therapy is often linked with side effects that are mostly related to the high dosage necessary because of relatively high degradation rate (proteolysis) and fast clearance (due to the initial inflammatory response). ${ }^{2,14,17}$ This demonstrates the need for a more reliable delivery system to ensure adequate protein concentration at the target sites. $2,14,18$

Our approach involves the adsorption of BMP-7 onto titanium surfaces coated with PEA and FN (organized into nanonetworks). This configuration allowed us to dramatically decrease the doses of BMP-7 used to stimulate functionalization of artificial implants, while keeping its osteogenic capabilities (Figure 1a). The target outcome would be a better/faster local response of hMSCs toward osteoblastic differentiation on this surface.

\section{MATERIALS AND METHODS}

2.1. Titanium Discs and Polymer Coating. Titanium discs were fabricated from Ti metal ( $99.995 \%$ purity, Pi-KEM Ltd.) deposited on $12 \mathrm{~mm}$ diameter glass coverslips using a Plassys MEB550S Electron Beam Evaporator (Kelvin Nanotechnology Ltd.). To reach a thickness of $750 \mathrm{~nm}$ of $\mathrm{Ti}$, the deposition rate was $0.3 \mathrm{~nm} / \mathrm{s}$. Poly(ethyl acrylate) (PEA) was synthesized and spin-coated onto the titanium discs following the protocol described by Vanterpool et al. ${ }^{7}$ Briefly, PEA sheets were prepared by radical polymerization of ethyl acrylate solutions using $1 \%$ benzoin as photoinitiator. Thin films were prepared by spin-coating PEA (2.5\%) solutions in toluene on Ti discs for $30 \mathrm{~s}$ at $2000 \mathrm{rpm}$. Before use, samples were oven-dried at $60^{\circ} \mathrm{C}$ and vacuum extracted.

2.2. Protein Coating. Protein coating of Ti discs was carried out with $20 \mu \mathrm{g} / \mathrm{mL}$ fibronectin isolated from human plasma (R\&D Systems Europe Ltd.). For atomic force microscopy (AFM) assays, the coating was performed for a time of $10 \mathrm{~min}$; then, samples were washed once with PBS and three times with ultrapure water. ${ }^{19}$ For all other experiments, titanium samples were UV-sterilized for $20 \mathrm{~min}$ and coated with $20 \mu \mathrm{g} / \mathrm{mL}$ FN solution in DPBS (Gibco, Life Technologies) for $1 \mathrm{~h} .{ }^{7,19}$ After that, the samples were washed with DPBS and then coated with $25 \mathrm{ng} / \mathrm{mL}$ or $100 \mathrm{ng} / \mathrm{mL}$ BMP-7 solutions in DPBS (human recombinant BMP-7 cat. no. 354-BP, R\&D Systems Europe Ltd.) for $1 \mathrm{~h}$. This was followed by a single wash with DPBS; GF-negative samples were treated with DBPS. The volume of coating solution and subsequent washes was $200 \mu \mathrm{L}$ per sample in all cases. After the coating, samples were preserved in DPBS until cell seeding.

2.3. Characterization Tests. 2.3.1. Surface Wettability and Homogeneity. Water contact angle (WCA) analysis was carried out on Ti discs, glass discs, and on PEA-coated surfaces of both $\mathrm{Ti}$ and glass discs using a THETA LITE optical tensiometer (Biolin Scientific, Stockholm, Sweden) according to the method described previously. a)

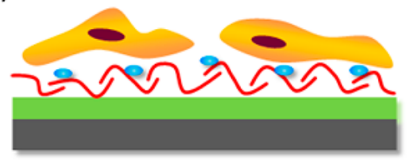
hMSC BMP-7 FN PEA $\mathrm{Ti}$

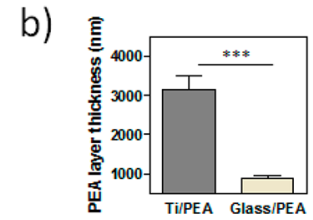

c)
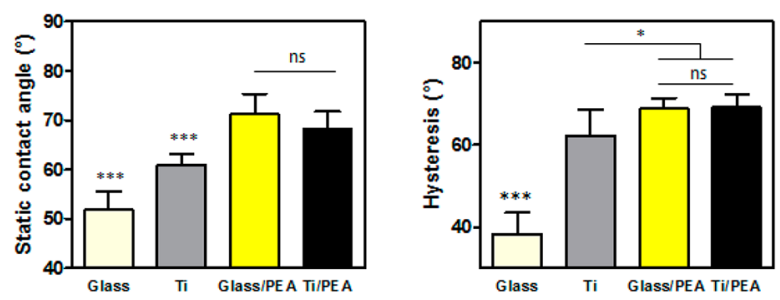

d)
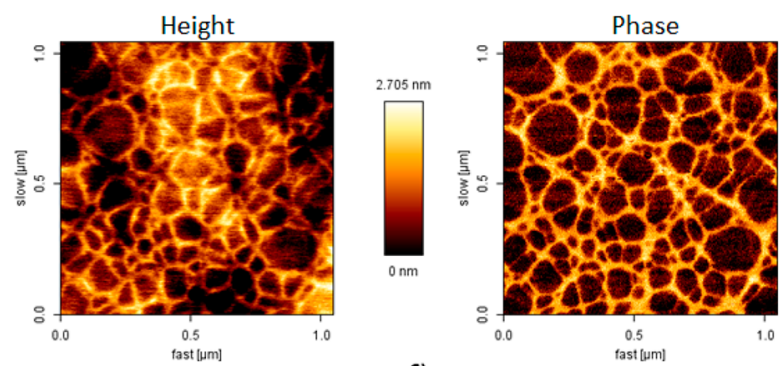

e)

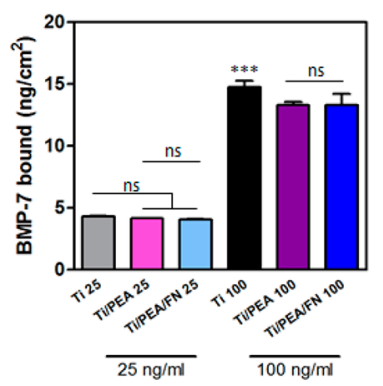

f)

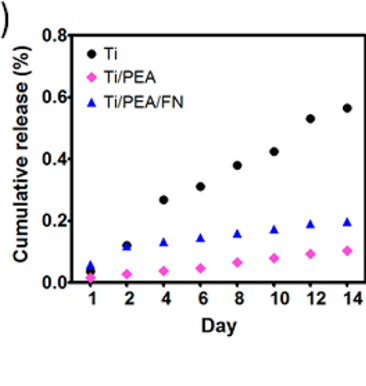

Figure 1. Characterization of engineered surfaces. (a) Scheme of a biomimetic system consisting of Ti layer coated with PEA functionalized with FN and specifically bound BMP-7 allowing synergistic signaling. (b) Thickness of polymer layer on $\mathrm{Ti}$ in comparison with glass tested by scratch assay; $* * * P<0.001$ (unpaired $t$ test was used). (c) Static water contact angle and hysteresis of bare Ti surface and $\mathrm{Ti} /$ PEA, in comparison with glass surfaces; $* P<0.05$; $* * * P<0.001$ (one-way ANOVA with Tukey's post-test was used). (d) AFM images of FN network on Ti/PEA surfaces. (e) BMP-7 bound to Ti, Ti/PEA, and Ti/PEA/FN at two BMP-7 concentrations; the total amount of BMP-7 bound per $\mathrm{cm}^{2}$ is shown; $\mathrm{ns}=$ nonsignificant; $* * * P<0.001$ (one-way ANOVA with Tukey's post-test was used). (f) Cumulative BMP-7 release from materials over 2 weeks; results shown as percentage from the BMP-7 amount originally bound after $1 \mathrm{~h}$ of coating. Asterisk labels above the bar without any linking lines to other bars means this condition was significantly different from all other conditions in the graph with the same level of significance.

For each condition, static contact angle (SCA), advancing contact angle (ACA), and receding contact angle (RCA) were determined. Eight different areas from two discs were analyzed for each angle value. Contact angle hysteresis was interpreted as a measure of molecular mobility for surfaces which are chemically homogeneous and flat. Hysteresis values were calculated for each tested drop using ACA and RCA measurements of that drop as a difference between ACA and RCA. $^{20}$

2.3.2. Atomic Force Microscopy (AFM). Ti discs coated with PEA (Ti/PEA) and FN (Ti/PEA/FN) were prepared. These discs were imaged using the AFM Nanowizard 3, JPK (Berlin, Germany). This method was used to demonstrate the arrangement of FN adsorbed on Ti surfaces. Three to six areas per sample were scanned (area size $(1 \times$ 
1) $\mu \mathrm{m}^{2}$ ) with a line rate $1.0 \mathrm{~Hz}$. The surface lock-in phase was observed in tapping-mode using a cantilever with a force constant of 3 $\mathrm{N} / \mathrm{m}$ and a resonance frequency of $75 \mathrm{kHz}$ (MPP-21120, Bruker, Billerica, MA). ${ }^{7}$ AFM imaging of uncoated Ti disc was performed using a cantilever with a force constant of $40 \mathrm{~N} / \mathrm{m}$ and a resonance frequency of $300 \mathrm{kHz}$ (DD-ACTA from AppNano). The AFM measured height images of $\mathrm{Ti}$ alone discs were then utilized as an indication of surface roughness. Nine scanned images with different scan areas $(1 \times 1$ to $30 \times 30 \mu \mathrm{m})$ were included. The profile average roughness values $\left(R_{\mathrm{a}}\right)$ and the root-mean-square roughness values $\left(R_{\mathrm{q}}\right)$ were calculated by the manufacturer software (JPK Data Processing). AFM was also used for measuring the thickness of the deposited $\mathrm{Ti}$ and PEA layers: $\mathrm{Ti}$ and $\mathrm{Ti} / \mathrm{PEA}$ discs were scratched to expose the underlying glass layer of the coverslip using a sharp razor ( 3 parallel scratch lines per disc in 2 replicates), height profiles were recorded across the scratch lines, and thicknesses of $\mathrm{Ti}$ and PEA layers were calculated.

2.3.3. BMP-7 Adsorption and Release. The amounts of BMP-7 bound to $\mathrm{Ti}, \mathrm{Ti} / \mathrm{PEA}$, and TI/PEA/FN surfaces were quantified by indirect enzyme-linked immunosorbent assay (ELISA) using DuoSet human BMP-7 kit (R\&D Systems, Minneapolis, MN). ${ }^{7}$ After the samples were coated with BMP-7, the coating solutions and the first wash solutions were collected for analysis. The amount of BMP-7 bound to the samples was calculated as a difference between the total BMP-7 used for coating and the amount of BMP-7 in the collected solution. Two concentrations of BMP-7, $25 \mathrm{ng} / \mathrm{mL}$ and $100 \mathrm{ng} / \mathrm{mL}$, were used for the initial coating; all samples were in triplicates. Cumulative BMP-7 release from all three tested surfaces was studied over 2-week period after initial coating with $25 \mathrm{ng} / \mathrm{mL}$ BMP-7 solution; samples were incubated at $37{ }^{\circ} \mathrm{C}$ and $5 \%$ of $\mathrm{CO}_{2}$ covered with $200 \mu \mathrm{L}$ of DPBS; solutions were collected on the day 1, 2 and then every second day, and the amount of BMP-7 released was assessed using the same sandwich ELISA as for the BMP-7 adsorption.

2.4. Cell Cultures. Human mesenchymal stem cells (hMSCs) were obtained from Promocell (cat. no. C-12975); cells were expanded in high-glucose DMEM (Invitrogen) supplemented with $1.0 \%$ penicillin/ streptomycin and 5\% FBS. Cells were passaged not more than 5 times throughout the experiments. Cells were cultured at $37^{\circ} \mathrm{C}, 5 \%$ of $\mathrm{CO}_{2}$, and $100 \%$ humidity in a HeraCell incubator.

2.4.1. hMSCs Proliferation and Mineralization. For the proliferation assay, cells were seeded on $\mathrm{Ti}, \mathrm{Ti} / \mathrm{BMP}-7, \mathrm{Ti} / \mathrm{PEA} / \mathrm{FN}$, and $\mathrm{Ti} /$ PEA/FN/BMP-7 surfaces $(25 \mathrm{ng} / \mathrm{mL}$ of BMP-7 was used for the GF coating where applicable). Seeding was done in serum-free medium (high-glucose DMEM supplemented with $1.0 \%$ penicillin/streptomycin) in 24-well plates at 10000 cells/well (4 replicates per each time point, day 1 and day 28 from seeding). After $2.5 \mathrm{~h}$, the media were changed to low serum growth medium (high-glucose DMEM supplemented with $1.0 \%$ penicillin streptomycin and $5.0 \% \mathrm{FBS}){ }^{15}$ Subsequently, the media were regularly changed every 2 days. An additional set of 4 samples with cells on $\mathrm{Ti}$ surface was kept in osteogenic medium (high-glucose DMEM supplemented with $10 \%$ FBS, $10^{-7} \mathrm{M}$ dexamethasone, $25 \mu \mathrm{g} / \mathrm{mL}$ L-ascorbic acid, and $3 \mathrm{mM}$ $\mathrm{NaH}_{2} \mathrm{PO}_{4}$ ), with the medium regularly changed. Cell proliferation was assessed by using alamarBlue cell viability assay (Invitrogen). ${ }^{21}$ Prior the assay, samples were transferred into a new 24-well plate and a fresh low serum nonosteogenic medium was added to all wells. alamarBlue reagent was then added at $50 \mu \mathrm{L} /$ well followed by incubation for exactly $2.5 \mathrm{~h}$ at $37^{\circ} \mathrm{C}$. Afterward, $100 \mu \mathrm{L}$ was transferred from each well into a black 96-well plate (Greiner Bio-One), and fluorescence was measured immediately at $590 \mathrm{~nm}$ (with excitation at $560 \mathrm{~nm}$ ) (Infinite M200 PRO plate reader, Tecan Group Ltd.). The media were then aspirated from the 24-well plate, and cells were washed twice with DBPS without calcium and magnesium (Gibco).

Matrix mineralization was determined by a direct assay of total calcium produced by the seeded cells under the same conditions as for the proliferation assay. Calcium amount was analyzed by complexing o-cresolphthalein complexone (Sigma-Aldrich, Fluka Analytical), 8Hydroxyquinoline (Sigma-Aldrich) and alkaline buffer reagent (SigmaAldrich), according to reported methods. ${ }^{22}$ A standard calibration curve was established to detect dilutions of $0-1600 \mu \mathrm{g} / \mathrm{mL} \mathrm{CaCl}{ }_{2}$.
$6 \mathrm{H}_{2} \mathrm{O}$ (Sigma-Aldrich). ${ }^{22}$ Measurements of calcium were performed according to the following protocol: $500 \mu \mathrm{L}$ of $1 \mathrm{M} \mathrm{HCl}$ was added per well to lyse the cells. The plate was sealed and left on a rocker at $100 \mathrm{rpm}$ for $8 \mathrm{~h}$ at room temperature to complete the lysation process. Samples were then collected, and the $o$-cresolphthalein complexone protocol was applied to obtain the calcium absorbance values which were then converted according to the $\mathrm{CaCl}_{2} \cdot 6 \mathrm{H}_{2} \mathrm{O}$ standard curve. ${ }^{22}$ Another standard calibration curve relating known seeding counts of hMSCs to the corresponding measured fluorescence (at excitation 560 $\mathrm{nm} /$ emission $590 \mathrm{~nm}$ ) was developed to interpret alamarBlue fluorescence readings. ${ }^{21}$ Calcium production results were then normalized to the obtained viable cell count for each disc and converted from $\mathrm{CaCl}_{2} \cdot 6 \mathrm{H}_{2} \mathrm{O}$ to $\mathrm{Ca}^{2+}$ equivalents and expressed in picograms (pg) per viable cell.

2.4.2. Cell Viability. A LIVE/DEAD Viability/Cytotoxicity Kit "for mammalian cells" (Molecular Probes) and NucBlue Live ReadyProbes Reagent (Molecular Probes) were used to assess cell viability. ${ }^{23}$ Passage $1 \mathrm{hMSCs}$ were seeded in 24-well plates for visualizing live and dead cells on the discs after 1 and 28 days of cell culture. The tested surfaces were seeded at 10000 cells/disc in duplicates for day 1 and in 4 replicates for day 28 . The medium and the seeding procedure were identical to the alamarBlue assay; media were changed every 2 days. At each time point (day 1 and day 28), a staining solution was made by adding $8 \mu \mathrm{L}$ of ethidium homodimer- 1 and $2 \mu \mathrm{L}$ of calcein $\mathrm{AM}$ to 90 $\mu \mathrm{L}$ of DPBS (Sigma-Aldrich), and $10 \mu \mathrm{L}$ of the staining solution per well was added, supplemented by $15 \mu \mathrm{L}$ of NucBlue solution (without removing the media). Samples were incubated $15 \mathrm{~min}$ at $37^{\circ} \mathrm{C}$, and then the discs were flipped inside the wells and directly imaged by fluorescent microscope (ZEISS AxioObserver.Z1).

2.4.3. Immunofluorescence Staining. For focal adhesions, hMSCs were seeded in a 24-well plate at a cell density of $3500 \mathrm{cells} / \mathrm{cm}^{2}$ onto following surfaces: Ti/PEA/FN/BMP-7, Ti/PEA/FN, Ti/BMP-7, and Ti only; samples were incubated for $24 \mathrm{~h}$, then washed with PBS, fixed with $4.0 \%$ formaldehyde, permeabilized and stained for vinculin using mouse monoclonal antivinculin antibody (Sigma-Aldrich) (1:400 dilution) detected by $\mathrm{Cy3}$-conjugated Affinipure rabbit antimouse IgG (Jackson ImmunoResearch Laboratories Inc., West Grove, PA) (1:200 dilution). Antibodies were diluted in 1.0\% BSA (SigmaAldrich) in PBS. Cytoskeleton was stained with BODIPY FL Phallacidin (Molecular Probes) (1:100 dilution in PBS), and samples were mounted with Vectashield with DAPI (Vector Laboratories).

A fluorescent microscope ZEISS AxioObserver.Z1 was used for imaging. Focal Adhesion Analysis Server (http://faas.bme.unc.edu/) ${ }^{24}$ was used for focal adhesion analysis of individual cells, and RStudio software (Version 0.98.1102, RStudio, Inc.) and "R script" (by Vanterpool and Gurden) were used to process the data obtained from the server.

For late osteogenic markers staining, hMSCs were seeded at passage 2 into four well plates; two for osteocalcin (day 1 and day 28) and two for osteopontin (day 1 and day 28). Four sample conditions were included (Ti/PEA/FN/BMP-7, Ti/PEA/FN, Ti/BMP-7, and Ti only), and the seeding density, cell culture steps, and culture medium were identical to alamarBlue. The design incorporated duplicates for day 1 and 4 replicates for day 28 in each group of surfaces. The media were changed every 2 days until the time of analysis. At each time point, the discs in the corresponding well plates were washed, fixed, and stained for osteopontin (1:50 dilution) and osteocalcin (1:50 dilution) (SantaCruz Biotechnologies). ${ }^{25}$ A 1:500 dilution of rhodamine phalloidin mixed with the secondary biotinylated antibody solution was prepared, and applied to the sample discs. After washing and mounting with Vectashield with DAPI, images were taken using three channels of a fluorescent microscope (osteocalcin or osteopontin (green), actin (red), and DNA (blue)). Image analysis and quantification of osteocalcin and osteopontin staining and number of nuclei was done using ImageJ software (Wayne Rasband, National institutes of Health, Bethesda, MD, version 1.49P).

2.5. Statistical Analysis. All experimental results were interpolated and analyzed using Microsoft Excel 2010 and GraphPad Prism (GraphPad Software Inc.). Means and standard deviations were calculated, and data were analyzed by $t$ test, one-way analysis of 
variance (ANOVA) test with Tukey's multiple comparison post-test, or two-way ANOVA test with Bonferroni post-test where applicable (mentioned in the text).

\section{RESULTS}

3.1. Characterization of Engineered Microenvironments. Titanium samples were spin-coated with PEA solution resulting in a PEA layer attached to Ti surface through physical interaction after solvent evaporation. Their characterization was done by testing the thickness of PEA coating (Figure $1 \mathrm{~b}$ ), and surface wettability and molecular mobility. Figure 1c shows that Ti coated with PEA has higher hydrophobicity in comparison with the bare Ti surface.

Differences in the surface molecular mobility (homogeneity) were reflected in the hysteresis results (Figure 1c). Uncoated $\mathrm{Ti}$ surfaces (hysteresis $\approx 59.92^{\circ}$ ) were significantly more homogeneous than PEA-coated ones (hysteresis $\approx 68.23^{\circ}$ ) (Figure 1c). The ability of PEA to induce FN assembly into nanonetwork was assayed by AFM; Figure 1d shows FN networks on Ti/PEA samples, while a scan of PEA-only-coated $\mathrm{Ti}$ surface showed only a homogeneous signal (Supporting Information (SI) Figure S1). The presence of FN network on the $\mathrm{Ti} / \mathrm{PEA}$ surface confirmed observations from other studies that FN conformation on PEA does not retain its globular shape typical for nonspecifically bound FN or FN in solution. FN network assembly occurs through physical adsorption on PEA with no covalent bonding as described earlier, ${ }^{10}$ allowing the encrypted domains for GFs and cell binding to be exposed. ${ }^{11}$ The uncoated Ti metal surface AFM scan is also shown (SI Figure S1). The mean average profile roughness values $\left(R_{\mathrm{a}}\right)$ was found to be $6.50 \pm 0.56 \mathrm{~nm}$, whereas the rootmean-square roughness $\left(R_{\mathrm{q}}\right)$ was $8.25 \pm 0.76 \mathrm{~nm}$.

Binding of BMP-7 was studied using $25 \mathrm{ng} / \mathrm{mL}$ and $100 \mathrm{ng} /$ $\mathrm{mL}$ concentrations of BMP-7. At a lower BMP-7 concentration in the coating solution, approximately $5 \mathrm{ng} / \mathrm{cm}^{2}$ of BMP-7 (more than $80 \%$ of the total protein) was bound to all sample types ( $\mathrm{Ti}, \mathrm{Ti} / \mathrm{PEA}, \mathrm{Ti} / \mathrm{PEA} / \mathrm{FN}$ ) (Figure 1e) with no significant differences found. Using a more concentrated BMP-7 coating solution, the amount of approximately $15 \mathrm{ng} /$ $\mathrm{cm}^{2}$ of BMP-7 was bound to the surfaces, which represents lower percentages in comparison with samples where $25 \mathrm{ng} /$ $\mathrm{mL}$ of BMP-7 was used for coating (SI Figure S2). However, no significant differences were found when comparing the BMP-7 amounts bound to Ti, Ti/PEA, and Ti/PEA/FN discs at the coating concentration that was used for all experiments with cells $(25 \mathrm{ng} / \mathrm{mL})$ (Figure 1e). This is important as differences in biological effects between $\mathrm{Ti}$ only and $\mathrm{Ti} / \mathrm{PEA} /$ FN cannot be ascribed to different amounts on BMP-7 adsorbed on these surfaces but rather to the level of specificity of this binding, with FN networks enabling specific noncovalent protein-protein interactions between BMP-7 and heparinbinding domain of FN (FNIII12-14). This brings BMP-7 to the close vicinity of the integrin-binding domain (FNIII9-10) and allows enhanced synergistic signaling toward differentiation after cell adhesion as it has been already described for BMP-2 and VEGF. ${ }^{11,12}$ We also monitored BMP-7 release from all three types of surfaces over a 2 -week period; in all conditions, we observed minimal and stable release in time (Figure 1f). From the day 4 until the end of the experiment, different conditions released significantly different amounts of BMP-7 when compared to each other with $\mathrm{Ti}$ surface releasing the highest amount and TI/PEA the lowest. The difference between Ti/PEA and TI/PEA/FN and thus differences in cell behavior afterward cannot be ascribed to different release rates between both systems. Overall, we observed that BMP-7 binding was very strong on all surfaces as not more than $1 \%$ of the BMP-7 bound to surfaces was released after 2 weeks.

3.2. Cell Adhesion. Analyses of focal adhesions (FA) and cell area were performed $24 \mathrm{~h}$ after cell seeding. These assays revealed that the size of hMSCs was similar on all surfaces (about $5000 \mu \mathrm{m}^{2}$, Figure 2a top). Measurements of total length
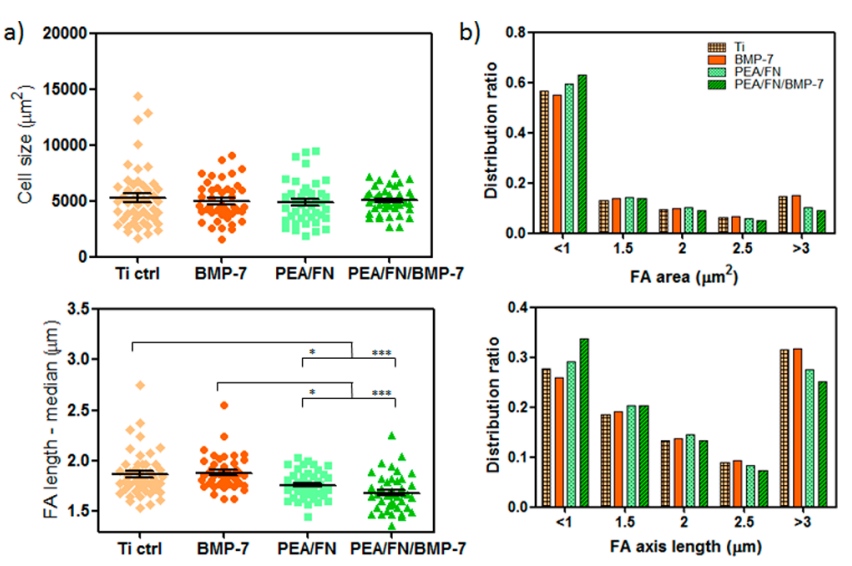

c)

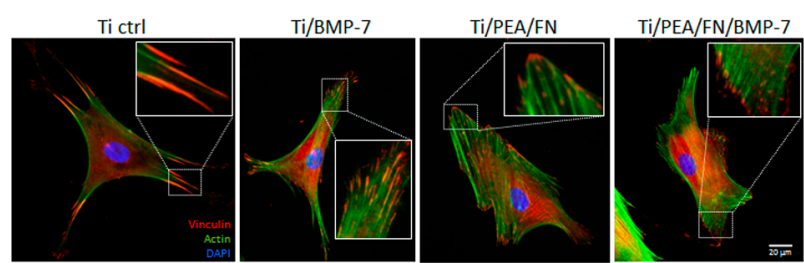

Figure 2. Focal adhesion (FA) analysis in hMSC on material surfaces: (a) Average cell size on $\mathrm{Ti}, \mathrm{Ti} / \mathrm{PEA}$ and with or without BMP-7 coating did not vary but significant differences were found for FA length- $\mathrm{Ti}$ with PEA coating had shorter $\mathrm{FA}$ than $\mathrm{Ti}$ alone or $\mathrm{Ti} /$ BMP-7; * $P<0.05$; *** $P<0.001$ (one-way ANOVA with Tukey's post-test was used). (b) Distribution ratios for FA area and FA length. (c) Representative images of fluorescence labeled hMSC: Shorter FA are present more often on PEA surfaces in comparison with $\mathrm{Ti}$ or $\mathrm{Ti} /$ BMP-7 (inserts); vinculin representing $\mathrm{FA}$ is stained in red, cytoskeleton in green, and nuclei in blue; scale bar is $20 \mathrm{um}$.

of focal adhesions showed that the total FA length was significantly higher for the bare $\mathrm{Ti}$ and $\mathrm{Ti} / \mathrm{BMP}-7$ in comparison with samples coated with PEA (Figure 2a bottom).

There was no difference found between the median of FA length neither for bare $\mathrm{Ti}$ and $\mathrm{Ti} / \mathrm{BMP}-7$ nor for Ti/PEA/FN and $\mathrm{Ti} / \mathrm{PEA} / \mathrm{FN} / \mathrm{BMP}-7$. More detailed FA analysis (see SI Figure S3 for details of image processing) showed that distribution ratios for FA area (Figure $2 \mathrm{~b}$ top) and distribution ratios for FA axis length (Figure $2 \mathrm{~b}$ bottom) correlate well. For example, in the group of focal adhesions larger than $3 \mu \mathrm{m}^{2}$ and longer than $3 \mu \mathrm{m}$, there are more focal adhesions for Ti only and $\mathrm{Ti} / \mathrm{BMP}-7$ samples, whereas in the group of the smallest and shortest focal adhesions, there is higher prevalence of these adhesions for $\mathrm{Ti} / \mathrm{PEA} / \mathrm{FN}$ and $\mathrm{Ti} / \mathrm{PEA} / \mathrm{FN} / \mathrm{BMP}-7$ samples. Representative fluorescence images showing vinculin present in focal adhesions (Figure 2c) illustrate the results by showing larger and longer focal adhesions on bare $\mathrm{Ti}$ surface and $\mathrm{Ti} /$ BMP-7 in comparison with Ti/PEA/FN and Ti/PEA/FN/ BMP-7 substrates. Very large and more developed $\mathrm{FAs}$ on $\mathrm{Ti}$ surfaces without PEA/FN coating suggest the cells are less mobile on these substrates while more typical FA signature 
found on Ti/PEA/FN and Ti/PEA/FN/BMP-7 surfaces implies the cell flexibility and higher potential for remodelling and differentiation.

3.3. Growth and Differentiation of hMSCs. Total cell numbers were quantified for day 1 and day 28 (Figure 3a). As
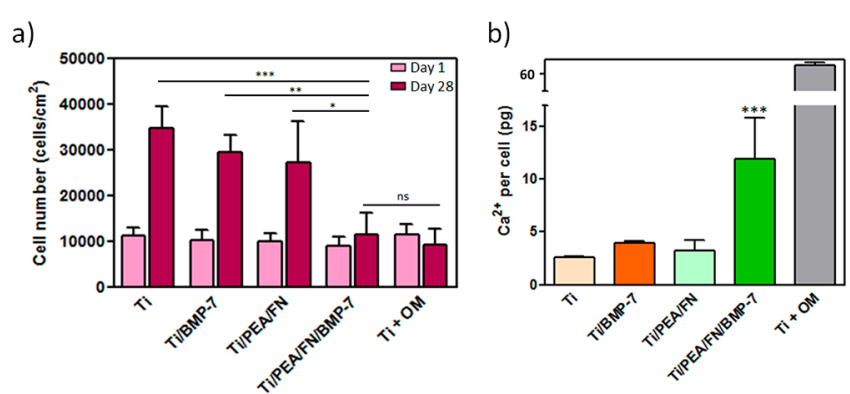

Figure 3. Cell proliferation and mineralization on Ti surfaces: (a) Cell numbers on $\mathrm{Ti} / \mathrm{PEA} / \mathrm{FN} / \mathrm{BMP}-7$ are significantly smaller in comparison to three control surfaces ( $\mathrm{Ti}$ alone, Ti/BMP-7, and $\mathrm{Ti} /$ PEA/FN) after 28 days (dark bars), corresponding to the differentiation process rather than cell proliferation; a positive differentiation control with hMSC growing on Ti surface in the presence of osteogenic medium $(\mathrm{Ti}+\mathrm{OM})$ also does not show any proliferation after 28 days; $* P<0.05$; ** $P<0.01$; *** $P<0.001$ (two-way ANOVA with Bonferroni post-test was used). (b) Free calcium production assay at day 28 showed higher amount of $\mathrm{Ca}^{2+}$ in hMSC on Ti surface with PEA/FN/BMP-7 coating (dark green bar) in comparison with other samples in growth medium, suggesting this system to have the best potential for mineralization; differences between other samples in growth medium were not significant; $* * * P$ $<0.001$ (one way ANOVA with Tukey's post-test was done). An asterisk label above the bar without any linking lines to other bars means this condition was significantly different from all other conditions in the graph with the same level of significance.

expected, there was no significant difference between the samples in number of cells 1 day after the seeding. After 28 days, the number of cells increased significantly for $\mathrm{Ti}, \mathrm{Ti} /$ BMP-7, and Ti/PEA/FN samples, whereas for Ti/PEA/FN/ BMP-7 samples, it remained at the same level as on the day $1-$ this is expected if cells are differentiating rather than proliferating. This result was supported by a lack of proliferation on a differentiating control sample (cells seeded on bare $\mathrm{Ti}$ and cultivated in osteogenic medium) on day 28 when compared with day 1 . The decreased rate of cell proliferation on Ti/PEA/FN/BMP-7 samples at the day 28 was also confirmed by additional cell number analysis based on quantification of nuclei on the tested surfaces (image analysis of DAPI staining); the results including a comparison with alamarBlue assay is shown in SI Figure S4. Live/Dead assay at day 28 confirmed that generally all cells found on surfaces were alive (SI Figure S5 shows representative fluorescence images for this assay on day 28 and day 1 ).

Cell differentiation into osteogenic lineage was monitored by measuring the level of calcium production, and also the expression of osteogenic markers osteocalcin (OCN) and osteopontin (OPN). Figure $3 \mathrm{~b}$ shows normalized free calcium production per viable cell for all tested surfaces and reveals the system as the one with the highest calcium level and thus with the best potential for osteogenic differentiation. Cells on the three other systems produced generally less than a half of the calcium amount than on Ti/PEA/FN/BMP-7 surface, and their calcium level values were not significantly different among each other. Osteogenic markers OCN and OPN were visualized in hMSC by green immunofluorescence after 28 days of cell culture in growth medium (Figure $4 \mathrm{a}$ ) and quantified by image analysis. OCN production was significantly higher for cells on Ti/PEA/FN/BMP-7 surface when compared with cells on bare $\mathrm{Ti}$ and on $\mathrm{Ti} / \mathrm{PEA} / \mathrm{FN}$ (Figure $4 \mathrm{~b}$ top). OPN expression was strongly enhanced in cells on Ti/PEA/FN/BMP-7 samples (Figure $4 \mathrm{~b}$ bottom); presence of OPN on the other three surfaces was lower and did not vary significantly among these three conditions (Figure $4 \mathrm{~b}$ bottom).

\section{DISCUSSION}

Titanium implants have shown long-term clinical success in single and multiple restorations; however, they face the disadvantage of prolonged osseointegration time. ${ }^{6,26}$ When titanium was used in combination with BMP-7 in the medium a)

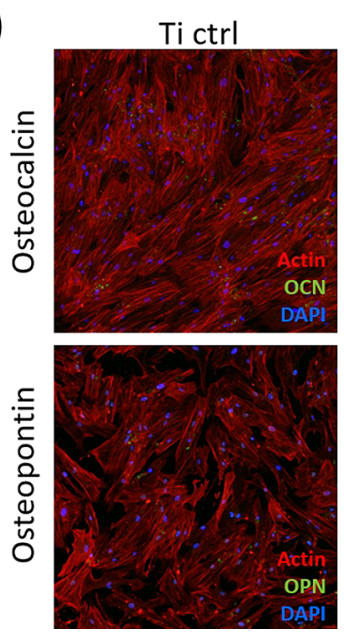

Ti/BMP-7
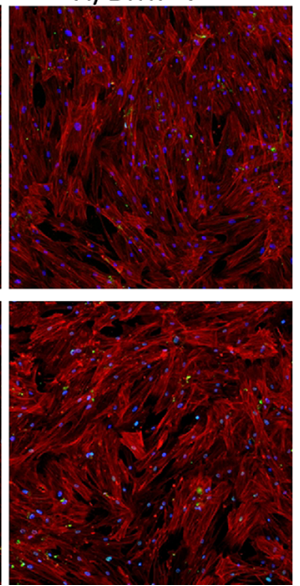

\section{Ti/PEA/FN}
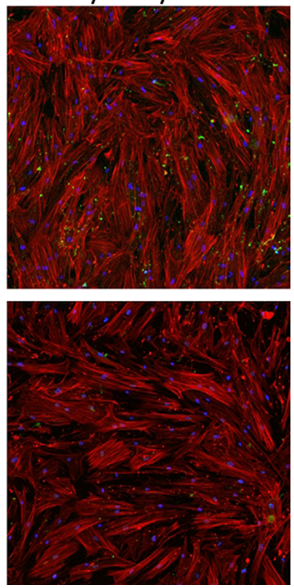
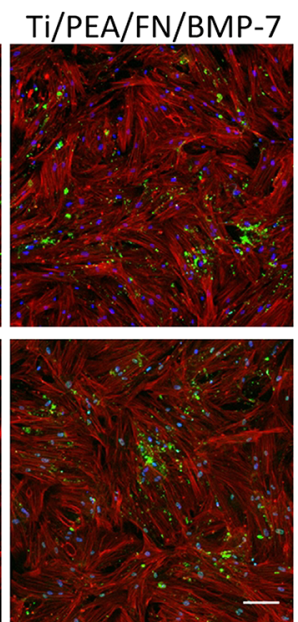

b)
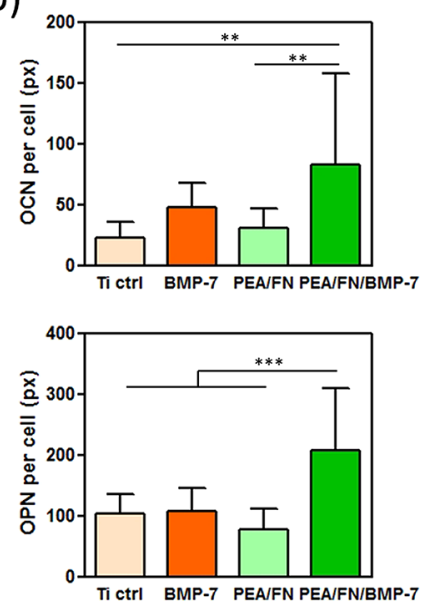

Figure 4. Osteogenic differentiation of hMSC on engineered surfaces after 28 days of culture: (a) Fluorescence images of hMSC with osteogenic markers osteocalcin (OCN, top), and osteopontin (OPN, bottom) on Ti-functionalized substrate (Ti/PEA/FN/BMP-7) and three control surfaces; OCN and OPN are stained in green, cytoskeleton is in red and DAPI in blue. Scale bar represents $100 \mu \mathrm{m}$. (b) Quantification of OCN and OPN staining showing a significant increase in both markers on Ti/PEA samples functionalized with FN and BMP-7 (dark green bars); $* * P<0.01$; $* * * P$ $<0.001$ (one-way ANOVA with Tukey's post-test was done). 
to study osteogenic effects in vitro, results were not uniform. For example, one study showed no benefit from the presence of soluble BMP-7, ${ }^{27}$ while other works suggested increased osteogenic potential in samples with BMP-7; ${ }^{28}$ however, continuous supply of the growth factor was necessary. To improve osteogenesis, while using low amounts of BMP-7, we coated Ti samples with PEA that induces FN arrangement into fibrillar nanonetworks to allow specific binding of BMP-7 to FN. ${ }^{7,10,29}$ Even though FN has been previously investigated as coating material for dental implants, no significant effects has been observed in vitro or in vivo. ${ }^{30-32}$ Corresponding to the important role of FN in ECM formation, FN nanonetworks on PEA have shown to promote cell adhesion and differentiation with different cell types, ${ }^{7,10,29}$ and more importantly, enhanced signaling via synergistic effect between FNIII9-10 (integrin binding region) and FNIII12-14 (GF binding domain) has been described using BMP-2 and VEGF bound to FN on PEA. ${ }^{11,12}$ In our Ti coatings, bound BMP-7 on assembled FN nanonetworks on $\mathrm{Ti} / \mathrm{PEA}$ activates growth factor receptors in concert integrins after cell attachment to FN network, and potentiates the response toward cell migration, proliferation and differentiation.

Other strategies to present growth factors on material surfaces include protein engineering techniques, the use of peptides that bind $\mathrm{GFs}^{33}$ and the use of layer-by-layer technologies. $^{34,35}$ For BMP-7, systems such as BMP-7-loaded PLGA nanocapsules in PCL scaffolds, ${ }^{36}$ BMP-7-adsorbed deproteinized bovine bone, ${ }^{37}$ BMP-7 loaded PLGA microspheres, ${ }^{38}$ microspheres from heparin-BMP-7 nanocomplex entrapped in polyester matrix, ${ }^{39}$ or cells producing BMP-7 through lentiviral transfection ${ }^{40}$ have been developed so far. However, these matrices all entrap BMP-7 nonspecifically, and then relatively high concentrations of the growth factor had to be used to achieve a biological effect. These strategies are in general more effective than the soluble administration of growth factors but, critically, do not exploit synergy between growth factor and integrin receptors to accelerate healing. ${ }^{41}$ The potential importance of crosstalk between integrins and growth factors was revealed in biological sciences more than a decade ago, ${ }^{42}$ and was explicitly engineered into a fibrin matrix using a recombinant fragment of FN consisting of FNIII9-10/ $12-14$ that promoted bone regeneration and wound healing. ${ }^{43}$ We have engineered the cellular microenvironment to target integrins and GF receptors synergistically with facile and translatable materials technology using a simple coating of PEA on Ti. Our system allows using much lower BMP-7 doses as it utilizes its unique property to display the bound GF efficiently next to the integrin binding domain and thus facilitates enhanced cellular signaling.

Because in this study FN is used for its specific conformation and ability to bind GFs prior cell attachment, for the final effect it is crucial to have FN adsorbed prior implantation. Nevertheless, we cannot exclude the role of interaction of PEA and FN that is naturally secreted by cells. Indeed, in our recent work we observed that although implantation of PEA coated with FN without BMP-2 did not bridge a critical size defect, it still provided higher levels of bone growth. ${ }^{11}$ We can speculate that cellular FN follows the cues given by PEA; however, considering the presence of other proteins, we do not expect the complete FN network would be formed. ${ }^{44}$

It has been reported that BMP-7 has the potential to alter the differentiation pathway in some cell types by hindering cell cycle progression during early cell differentiation. ${ }^{44}$ Our results corroborate these observations when presented from PEA surfaces, with the proliferation rate being reduced with the onset of differentiation. ${ }^{45}$ However, it has been reported that the proliferative effect of BMP-7 highly depends on the cell type and culture conditions. In several reports, it has been shown that hMSCs are nonresponsive to the induction of cellular proliferation upon BMP-7 supplementation, having a low level of mitogenic activity on these cells. ${ }^{8,15,45}$ Thus, our results are highly consistent with these reports, which confirms the osteogenic potential of BMP-7 presented from a solid phase on PEA at low concentrations. ${ }^{15,45}$

The mineralization of the bone adjacent to the implant surface is a crucial process in the stability of osseointegrated implants. ${ }^{26}$ Here, a direct assay of total calcium produced by the cells was applied. ${ }^{22,46}$ It has been reported that metal ions from the $\mathrm{Ti}$ surface potentially inhibit formation of hydroxyapatite crystals on $\mathrm{CP} \mathrm{Ti}$ or $\mathrm{Ti} 6 \mathrm{Al} 4 \mathrm{~V}$ surfaces in vivo. ${ }^{26}$ This could explain low levels of calcium production after 28 days for bare Ti surface and Ti/BMP-7. From our results, it is clear that the low concentration of BMP-7 used here is not high enough to trigger osteogenesis on bare $\mathrm{Ti}$, and about 10 times higher dose continuously supplied in the medium is needed as reported before ${ }^{8,15}$ while the same low BMP-7 amount $(25 \mathrm{ng} / \mathrm{mL}$ in a single coating) was sufficient for reaching both higher calcium levels and significant osteogenic differentiation when presented on our Ti/PEA/FN/BMP7 system. Therefore, BMP-7 bound to PEA/FN is able to promote cell differentiation using ultralow dose of BMP-7, as it reported previously for this polymer using BMP-2 and VEGF due to the crosstalk between integrins and GF receptors. ${ }^{11,12}$

There is a whole range of markers that can be used for assessment of the level of osteogenic differentiation in vitro including an early marker Runx2, osteocalcin, osteonectin, osteopontin or alkaline phosphatase assay. ${ }^{47}$ We are confident that by selecting osteocalcin and osteopontin protein expression together with calcium production assay, we can clearly show the osteogenic potential of our Ti/PEA/FN/BMP7 system as an increase of these parameters represents well the differentiation process progressing into later stages.

It is important to note that our results clearly showed that cell differentiation is predominantly due to the presence of PEA/FN/BMP7 instead of other properties exhibited by titanium surface, such as wettability or surface elasticity. Further work will be focused on the development of plasma polymerization of PEA enabling deposition of controlled thickness on the surface of complex 3D titanium implants that would be the next step toward clinical translation. Another translation-related question is linked to a potential foreign body reaction (FBR). We hypothesize that considering bioinert characteristics of titanium together with our previous in vivo studies with PEA not showing any major inflammatory response, FBR will be low. However, it remains to be assessed in future in vivo experiments. Stability of the Ti/PEA interface is also of an interest from a long-term perspective, particularly to avoid delamination and facilitating adhesion of the newly mineralized tissue. We expect that, in vivo, Ti/PEA/FN/BMP-7 will boost stem cells toward osteogenic differentiation but the thin layer of PEA will be eventually remodelled and metabolized by cells. For this, further long-term in vivo experiments need to be performed. 


\section{CONCLUSIONS}

The proposed delivery system for BMP-7 consisting of titanium surface coated with poly(ethyl acrylate) specifically adsorbs fibronectin exposing growth factor and integrin binding domains on its network. This strategy allowed us to functionalize these substrates with extremely low concentrations of BMP-7, and thus to create a microenvironment promoting hMSC differentiation toward osteogenic lineage. In vitro cellular experiments using hMSCs from bone marrow demonstrated the biocompatibility of the system and its ability to induce a better late osteogenic differentiation response in these cells. After 28 days of cell culture, the system induced the highest level of matrix mineralization of hMSCs compared to the control groups with identical cell culture media. This technology opens up the way to use very low locally delivered doses of BMP-7 to maximize osseointegration and thus increase implants safety.

\section{ASSOCIATED CONTENT}

\section{S Supporting Information}

The Supporting Information is available free of charge on the ACS Publications website at DOI: 10.1021/acsbiomaterials.7b01037.

AFM images of Ti surfaces, a scheme of focal adhesion analysis process, and fluorescence images of Live/Dead assay (PDF)

\section{AUTHOR INFORMATION}

\section{Corresponding Author}

*E-mail: Manuel.Salmeron-Sanchez@glasgow.ac.uk. ORCID $\odot$

Vladimíra Moulisová: 0000-0003-0700-2837

Matthew J. Dalby: https://orcid.org/0000-0002-0528-3359

Manuel Salmerón-Sánchez: 0000-0002-8112-2100

\section{Author Contributions}

II(M.A.-J., V.M.) These authors contributed equally. The manuscript was written through contributions of all authors.

\section{Notes}

The authors declare no competing financial interest.

Data availability: All the original data related to this article are within the depository of the University of Glasgow DOI: 10.5525/gla.researchdata.609.

\section{ACKNOWLEDGMENTS}

We thank Annie Zhe Cheng (School of Engineering, University of Glasgow) for preparing the titanium discs, and Frankie Vanterpool (Biomedical Engineering, University of Glasgow) and Ross Gurden (Centre for Cell Engineering, University of Glasgow) for developing, testing and introducing "R script" for focal adhesion analysis. This study was supported by the European Research Council (ERC 306990), the U.K. Regenerative Medicine Platform (MRC grant MR/L022710/ 1), and an EPSRC Programme Grant (EP/P001114/1).

\section{REFERENCES}

(1) Urist, M. R. Bone - formation by autoinduction. Science 1965, 150 (3698), 893.

(2) Carreira, A. C.; Lojudice, F. H.; Halcsik, E.; Navarro, R. D.; Sogayar, M. C.; Granjeiro, J. M. Bone Morphogenetic Proteins Facts, Challenges, and Future Perspectives. J. Dent. Res. 2014, 93 (4), 335345 .
(3) German, M.; Osei-Bempong, C.; Knuth, C.; Deehan, D.; Oldershaw, R. Investigating the biological response of human mesenchymal stem cells to titanium surfaces. J. Orthop. Surg. Res. 2014, 9 (1), 135.

(4) Santander, S.; Alcaine, C.; Lyahyai, J.; Perez, M. A.; Rodellar, C.; Doblare, M.; Ochoa, I. In vitro osteoinduction of human mesenchymal stem cells in biomimetic surface modified titanium alloy implants. Dent. Mater. J. 2014, 33 (3), 305-12.

(5) Thakral, G.; Thakral, R.; Sharma, N.; Seth, J.; Vashisht, P. Nanosurface - The Future of Implants. J. Clin. Diagn. Res. 2014, 8 (5), ZE07-ZE10.

(6) Yeo, I.-S. Reality of Dental Implant Surface Modification: A Short Literature Review. Open Biomed. Eng. J. 2014, 8, 114.

(7) Vanterpool, F. A.; Cantini, M.; Seib, F. P.; Salmeron-Sanchez, M. A material-based platform to modulate fibronectin activity and focal adhesion assembly. BioRes. Open Access 2014, 3 (6), 286-96.

(8) Zhang, F.; Ren, L. F.; Lin, H. S.; Yin, M. N.; Tong, Y. Q.; Shi, G. $\mathrm{S}$. The optimal dose of recombinant human osteogenic protein-1 enhances differentiation of mouse osteoblast-like cells: an in vitro study. Arch. Oral Biol. 2012, 57 (5), 460-8.

(9) Mouw, J. K.; Ou, G.; Weaver, V. M. Extracellular matrix assembly: a multiscale deconstruction. Nat. Rev. Mol. Cell Biol. 2014, 15 (12), 771-785.

(10) Salmeron-Sanchez, M.; Rico, P.; Moratal, D.; Lee, T. T.; Schwarzbauer, J. E.; Garcia, A. J. Role of material-driven fibronectin fibrillogenesis in cell differentiation. Biomaterials 2011, 32 (8), 2099105 .

(11) Llopis-Hernandez, V.; Cantini, M.; Gonzalez-Garcia, C.; Cheng, A. Z.; Yang, J.; Tsimbouri, P. M.; Garcia, A. J.; Dalby, M. J.; SalmeronSanchez, M. Material-driven fibronectin assembly for high-efficiency presentation of growth factors. Sci. Adv. 2016, 2, e1600188.

(12) Moulisova, V.; Gonzalez-Garcia, C.; Cantini, M.; RodrigoNavarro, A.; Weaver, J.; Costell, M.; Sabater i Serra, R; Dalby, M. J.; Garcia, A. J.; Salmeron-Sanchez, M. Engineered microenvironments for synergistic VEGF - Integrin signalling during vascularization. Biomaterials 2017, 126, 61-74.

(13) Rahman, M. S.; Akhtar, N.; Jamil, H. M.; Banik, R. S.; Asaduzzaman, S. M. TGF-beta/BMP signaling and other molecular events: regulation of osteoblastogenesis and bone formation. Bone Res. 2015, 3, Article No. 15005 DOI: 10.1038/boneres.2015.5.

(14) Chen, S.; Yang, J.; Wang, H.; Chao, Y.; Zhang, C.; Shen, J.; Zhang, P. Adenovirus encoding BMP-7 immobilized on titanium surface exhibits local delivery ability and regulates osteoblast differentiation in vitro. Arch. Oral Biol. 2013, 58 (9), 1225-31.

(15) Shen, B.; Wei, A.; Whittaker, S.; Williams, L. A.; Tao, H.; Ma, D. D.; Diwan, A. D. The role of BMP-7 in chondrogenic and osteogenic differentiation of human bone marrow multipotent mesenchymal stromal cells in vitro. J. Cell. Biochem. 2010, 109 (2), 406-416.

(16) Naudi, K. B.; Ayoub, A.; McMahon, J.; Di Silvio, L.; Lappin, D.; Hunter, K. D.; Barbenel, J. Mandibular reconstruction in the rabbit using beta-tricalcium phosphate (beta-TCP) scaffolding and recombinant bone morphogenetic protein 7 (rhBMP-7) - Histological, radiographic and mechanical evaluations. J. Cranio-MaxilloFac. Surg. 2012, 40 (8), E461-E469.

(17) Courvoisier, A.; Sailhan, F.; Laffenetre, O.; Obert, L. Bone morphogenetic protein and orthopaedic surgery: Can we legitimate its off-label use? Int. Orthop. 2014, 38 (12), 2601-2605.

(18) Lee, Y. J.; Lee, J.-H.; Cho, H.-J.; Kim, H. K.; Yoon, T. R.; Shin, $\mathrm{H}$. Electrospun fibers immobilized with bone forming peptide-1 derived from BMP7 for guided bone regeneration. Biomaterials 2013, 34 (21), 5059-5069.

(19) Gugutkov, D.; Altankov, G.; Rodriguez Hernandez, J. C.; Monleon Pradas, M.; Salmeron Sanchez, M. Fibronectin activity on substrates with controlled -OH density. J. Biomed. Mater. Res., Part A 2010, 92A (1), 322-331.

(20) Park, A.; Cima, L. G. In vitro cell response to differences in poly-L-lactide crystallinity. J. Biomed. Mater. Res. 1996, 31 (1), 117130. 
(21) Anoopkumar-Dukie, S.; Carey, J. B.; Conere, T.; O’Sullivan, E.; van Pelt, F. N.; Allshire, A. Resazurin assay of radiation response in cultured cells. Br. J. Radiol. 2005, 78 (934), 945-947.

(22) Holtorf, H. L.; Datta, N.; Jansen, J. A.; Mikos, A. G. Scaffold mesh size affects the osteoblastic differentiation of seeded marrow stromal cells cultured in a flow perfusion bioreactor. J. Biomed. Mater. Res., Part A 2005, 74A (2), 171-180.

(23) Haugland, R. P.; Maccoubrey, I. C.; Moore, P. L. Dualfluorescence cell viability assay using ethidium homodimer and calcein AM. U.S. Patent US5314805A, May 24, 1994.

(24) Berginski, M. E.; Gomez, S. M. The Focal Adhesion Analysis Server: a web tool for analyzing focal adhesion dynamics. F1000Research 2013, 2, 68-68.

(25) Gonzalez-Garcia, C.; Moratal, D.; Oreffo, R. O. C.; Dalby, M. J.; Salmeron-Sanchez, M. Surface mobility regulates skeletal stem cell differentiation. Integr. Biol. 2012, 4 (5), 531-539.

(26) Esposito, M.; Hirsch, J. M.; Lekholm, U.; Thomsen, P. Biological factors contributing to failures of osseointegrated oral implants - (II). Etiopathogenesis. Eur. J. Oral Sci. 1998, 106 (3), 721764.

(27) Togashi, A. Y.; Cirano, F. R.; Marques, M. M.; Pustiglioni, F. E.; Lang, N. P.; Lima, L. A. Effect of recombinant human bone morphogenetic protein-7 (rhBMP-7) on the viability, proliferation and differentiation of osteoblast-like cells cultured on a chemically modified titanium surface. Clin Oral Implants Res. 2009, 20 (5), 4527.

(28) Cirano, F. R.; Togashi, A. Y.; Marques, M. M.; Pustiglioni, F. E.; Lima, L. A. Role of rhBMP-2 and rhBMP-7 in the metabolism and differentiation of osteoblast-like cells cultured on chemically modified titanium surfaces. J. Oral Implant. 2014, 40 (6), 655-659.

(29) Rico, P.; Gonzalez-Garcia, C.; Petrie, T. A.; Garcia, A. J.; Salmeron-Sanchez, M. Molecular assembly and biological activity of a recombinant fragment of fibronectin (FNIII(7-10)) on poly(ethyl acrylate). Colloids Surf., B 2010, 78 (2), 310-6.

(30) Elkarargy, A. Biological functionalization of dental implants with fibronectin: a scanning electron microscopic study. Int. J. Health Sci.. (Qassim) 2014, 8 (1), 57-66.

(31) Lee, J.-S.; Yang, J.-H.; Hong, J.-Y.; Jung, U.-W.; Yang, H.-C.; Lee, I.-S.; Choi, S.-H. Early bone healing onto implant surface treated by fibronectin/oxysterol for cell adhesion/osteogenic differentiation: in vivo experimental study in dogs. J. Periodontal Implant Sci. 2014, 44 (5), 242-250.

(32) Brigaud, I.; Agniel, R.; Leroy-Dudal, J.; Kellouche, S.; Ponche, A.; Bouceba, T.; Mihailescu, N.; Sopronyi, M.; Viguier, E.; Ristoscu, C.; Sima, F.; Mihailescu, I. N.; Carreira, A. C. O.; Sogayar, M. C.; Gallet, O.; Anselme, K. Synergistic effects of BMP-2, BMP-6 or BMP-7 with human plasma fibronectin onto hydroxyapatite coatings: A comparative study. Acta Biomater. 2017, 55, 481-492.

(33) Hudalla, G. A.; Kouris, N. A.; Koepsel, J. T.; Ogle, B. M.; Murphy, W. L. Harnessing endogenous growth factor activity modulates stem cell behavior. Integr. Biol. 2011, 3 (8), 832-842.

(34) Martino, M. M.; Briquez, P. S.; Guc, E.; Tortelli, F.; Kilarski, W. W.; Metzger, S.; Rice, J. J.; Kuhn, G. A.; Muller, R.; Swartz, M. A.; Hubbell, J. A. Growth Factors Engineered for Super-Affinity to the Extracellular Matrix Enhance Tissue Healing. Science 2014, 343 (6173), 885-888.

(35) Crouzier, T.; Fourel, L.; Boudou, T.; Albiges-Rizo, C.; Picart, C. Presentation of BMP-2 from a Soft Biopolymeric Film Unveils its Activity on Cell Adhesion and Migration. Adv. Mater. 2011, 23 (12), H111-H118.

(36) Yilgor, P.; Hasirci, N.; Hasirci, V. Sequential BMP-2/BMP-7 delivery from polyester nanocapsules. J. Biomed. Mater. Res., Part A 2010, 93 A (2), 528-536.

(37) Liu, N.; Wang, Z. Sequential delivery of BMP-7 and IGF-I to enhance the osteoinductive property of deproteinized bovine bone. RSC Adv. 2016, 6 (55), 50322-50330.

(38) Gavenis, K.; Klee, D.; Pereira-Paz, R. M.; von Walter, M.; Mollenhauer, J.; Schneider, U.; Schmidt-Rohlfing, B. BMP-7 loaded microspheres as a new delivery system for the cultivation of human chondrocytes in a collagen type-I gel. J. Biomed. Mater. Res., Part B 2007, 82B (2), 275-283.

(39) Reguera-Nunez, E.; Roca, C.; Hardy, E.; de la Fuente, M.; Csaba, N.; Garcia-Fuentes, M. Implantable controlled release devices for BMP-7 delivery and suppression of glioblastoma initiating cells. Biomaterials 2014, 35 (9), 2859-2867.

(40) Chitty, D. W.; Tremblay, R. G.; Ribecco-Lutkiewicz, M.; Haukenfrers, J.; Zurakowski, B.; Massie, B.; Sikorska, M.; BaniYaghoub, M. Development of BMP7-producing human cells, using a third generation lentiviral gene delivery system. J. Neurosci. Methods 2012, 205 (1), 17-27.

(41) Martino, M. M.; Briquez, P. S.; Maruyama, K.; Hubbell, J. A. Extracellular matrix-inspired growth factor delivery systems for bone regeneration. Adv. Drug Delivery Rev. 2015, 94, 41-52.

(42) Comoglio, P. M.; Boccaccio, C.; Trusolino, L. Interactions between growth factor receptors and adhesion molecules: breaking the rules. Curr. Opin. Cell Biol. 2003, 15 (5), 565-571.

(43) Martino, M. M.; Tortelli, F.; Mochizuki, M.; Traub, S.; BenDavid, D.; Kuhn, G. A.; Mueller, R.; Livne, E.; Eming, S. A.; Hubbell, J. A. Engineering the Growth Factor Microenvironment with Fibronectin Domains to Promote Wound and Bone Tissue Healing. Sci. Transl. Med. 2011, 3 (100), 100ra89.

(44) Salazar, V. S.; Gamer, L. W.; Rosen, V. BMP signalling in skeletal development, disease and repair. Nat. Rev. Endocrinol. 2016, 12 (4), 203-21.

(45) Lavery, K.; Hawley, S.; Swain, P.; Rooney, R.; Falb, D.; AlaouiIsmaili, M. H. New insights into BMP-7 mediated osteoblastic differentiation of primary human mesenchymal stem cells. Bone 2009, 45 (1), 27-41.

(46) Kim, S. E.; Rha, H. K.; Surendran, S.; han, C. W. H.; Lee, S. C.; Choi, H. W.; Choi, Y.-W.; Lee, K.-H.; Rhie, J. W.; Ahn, S. T. Bone morphogenic protein-2 (BMP-2) immobilized biodegradable scaffolds for bone tissue engineering. Macromol. Res. 2006, 14 (5), 565-572.

(47) Graneli, C.; Thorfve, A.; Ruetschi, U.; Brisby, H.; Thomsen, P.; Lindahl, A.; Karlsson, C. Novel markers of osteogenic and adipogenic differentiation of human bone marrow stromal cells identified using a quantitative proteomics approach. Stem Cell Res. 2014, 12 (1), 15365. 